Pacific Journal of Mathematics

PS WTH -DDMENSIONAL CRTICAL 


\title{
MAPS WITH 0-DIMENSIONAL CRITICAL SET
}

\author{
P. T. Church and J. G. Timourian
}

Let $f: M^{n} \rightarrow N^{p}$ be $C^{n}$ with $n-p=0$ or 1 , let $p \geqq 2$, and let $R_{p-1}(f)$ be the critical set of $f$. If $\operatorname{dim}\left(R_{p-1}(f)\right) \leqq 0$, then (1.1) at each $x \in M^{n}, f$ is locally topologically equivalent to one of the following maps:

(a) the projection map $\rho: R^{n} \rightarrow R^{p}$,

(b) $\sigma: C \rightarrow C$ defined by $\sigma(z)=z^{d}(d=2,3, \cdots)$, where $C$ is the complex plane, or

(c) $\tau: C \times C \rightarrow C \times R$ defined by $\tau(z, w)=\left(2 z \cdot \bar{w},|w|^{2}-|z|^{2}\right)$, where $\bar{w}$ is the complex conjugate of $w$.

Under the additional hypothesis that $\operatorname{dim}\left(f\left(R_{p-1}(f)\right)\right) \leqq$ $p-2$ this result was proved in an earlier paper of the authors. They show here that $\operatorname{dim}\left(R_{p-1}(f)\right) \leqq 0$ implies something like $\operatorname{dim}\left(f\left(R_{p-1}(f)\right)\right) \leqq p-2$.

For general background material, the reader is referred to that earlier paper [5]. The branch set $B_{f}[5$, p. $616,(1.5)]$ is the set of points at which $f$ fails to be locally topologically equivalent to $\rho$. A map $g: J^{n-m} \times R^{m} \rightarrow L^{p-m} \times R^{m}$ is called a layer map if for each $t \in R^{m}, g\left(J^{n-m} \times\{t\}\right) \subset L^{p-m} \times\{t\}$.

1.2. Outline of the proof. We suppose that $f$ is not an open map, and from some technical differential lemmas of $\S 3$ obtain in (3.4) by restriction and change of coordinates a layer map satisfying the hypotheses of (2.1). By that lemma $\operatorname{dim}\left(B_{f}\right)=p-1$, so that $\operatorname{dim}\left(R_{p-1}(f)\right)=p-1$, contradicting the hypothesis of (1.1). Thus $f$ is open, and from the local structure for open maps given in [7] we conclude in (4.1) that $\operatorname{dim}\left(f\left(B_{f}\right)\right) \leqq p-2$. This is (essentially) the additional hypothesis assumed in [5], and our conclusion results. A global structure theorem is also given (4.5).

2. A topological lemma. In order to read the proof of (2.1) the reader will need to have at hand the definition and certain properties of spoke sets [7, (2.1), (2.2), (2.3)].

LEMma 2.1. Let $f: D^{2} \times R^{p-1} \rightarrow R \times R^{p-1}$ be a layer map with $B_{f} \neq \varnothing, f\left(\partial D^{2} \times\{t\}\right)$ a single point not in $f\left(B_{f}\right)$, and $\operatorname{dim}\left(B_{f} \cap\left(D^{2} \times\right.\right.$ $\{t\}))=\operatorname{dim}\left(f\left(B_{f} \cap\left(D^{2} \times\{t\}\right)\right)\right) \leqq 0$ for each $t \in R^{p-1}$. Then $\operatorname{dim} B_{f}=$ $p-1$.

Proof. The last hypothesis implies that $\operatorname{dim} f\left(B_{f}\right) \leqq p-1[9, \mathrm{p}$. 
44, Theorem IV 3], so that $\operatorname{dim} B_{f} \leqq p-1$ [9, p. 91, Theorem VI 7]. If $p=1$ and $B_{f}=\varnothing$, then $f$ is open and a contradiction results from [7, (3.1)(b) or (d)]. Thus, for $p=1 \operatorname{dim} B_{f}=0$, i.e., $p-1$. Hence we may suppose that $p \geqq 2$, and will prove that $\operatorname{dim} B_{f} \geqq$ $p-1$.

Let $I=[0,1]$, let $I^{p-1} \subset I^{p}$ be $\left\{\left(x_{1}, x_{2}, \cdots, x_{p}\right): x_{p}=0\right\}$, let $r=0,1$, $\cdots, p-1$, and, for $a \in I^{p-1}$, let $\Gamma_{a, r}=\left\{x \in I^{p-1}: x_{i}=a_{i}\right.$ for $\left.i \geqq r+1\right\}$. For

$$
X \subset \Gamma_{a, r} \text { and } \alpha>0,
$$

let $X(r, \alpha)=\left\{x \in I^{p-1}:\left(x_{1}, \cdots, x_{r}, a_{r+1}, \cdots, a_{p-1}\right) \in X\right.$ and $\left|x_{i}-a_{i}\right|<\alpha$ for $i \geqq r+1\}$. Thus $\Gamma_{a, r}(r, \alpha)=\left\{x \in I^{p-1}:\left|x_{i}-a_{i}\right|<\alpha\right.$ for $\left.i \geqq r+1\right\}$.

Consider statement $S_{r}:$ (1) for every $\varepsilon>0$ and $a \in I^{p-1}$, there are a triangulation $\mathfrak{T}$ of the $r$-cell $\Gamma_{a, r}$ and $\alpha>0$, and (2) for every closed $r$-simplex $\sigma$ of $\mathfrak{I}$, there are spoke sets $L_{j, \sigma}(j=0,1, \cdots, q(\sigma))$ satisfying conclusions (i)-(vi) of [7, (2.1) and (2.2)] with $W$ replaced by $\mathrm{Cl}[\sigma(r, \alpha)]$ and $E=B_{f} \cap\left(D^{2} \times I^{p-1}\right)$. Moreover, (3) let $\sigma$ and $\tau$ be closed $r$-simplices of $\mathfrak{T}$, and let $D^{2} \times \mathrm{Cl}[(\sigma \cap \tau)(r, \alpha)]$ be denoted by $T$. Then, for any $L_{i, \sigma}$ and $L_{j, \tau}$, one of the following statements is true: $L_{i, \sigma} \cap T=L_{j, \tau} \cap T, L_{i, \sigma} \cap T \subset\left(L_{j, \tau}-\Omega_{j, \tau}\right) \cap T, L_{j, \tau} \cap T \subset\left(L_{j, \sigma}-\right.$ $\left.\Omega_{j, \sigma}\right) \cap T$, or $L_{i, \sigma} \cap\left(L_{j, \tau}-\Omega_{j, \tau}\right) \cap T=\varnothing$.

Since $\Gamma_{a, 0}=\{a\}$ and $\{a\}$ is the only 0 -simplex of $T$, statement $S_{0}$ follows immediately from [7, (2.2)]. We will suppose that $S_{r}$ is true $(r<p-1)$ and deduce $S_{r+1}$.

Let $\varepsilon>0$ and $a \in I^{p-1}$ be given. For $[u, v] \subset R$ and $\eta>0$, let

$$
\Psi(u, v, \eta)=\left\{x \in I^{p-1}: u<x_{r+1}<v \text { and }\left|x_{i}-a_{i}\right|<\eta \text { for } i>r+1\right\} \text {. }
$$

If $c \in \Gamma_{a, r+1}$, then $\Gamma_{c, r} \subset \Gamma_{a, r+1}$ and $\Gamma_{c, r}(r, \eta)=\Psi\left(c_{r+1}-\eta, c_{r+1}+\eta, \eta\right)$. For $c \in \Gamma_{a, r+1}$, let $\alpha(c)>0, \mathfrak{T}(c)$, and $\left\{L_{c, j, o}\right\}$ be as given by $S_{r}$ for $\varepsilon$ (and $a$ replaced by $c)$. There are $c(i)(i=1,2, \cdots, m)$ such that $\left\{\Gamma_{c(i), r}(r\right.$, $\left.\left.\alpha\left(c_{i}\right)\right)\right\}$ covers $\Gamma_{a, r+1}$. We may suppose that $\left\{c_{r+1}(i)\right\}$ are in increasing order and the cover is minimal. If the open interval $\left(c_{r+1}(i)-\alpha(c(i))\right.$, $c_{r+1}(i)+\alpha(c(i))$ is denoted by $A_{i}$, then $0 \in A_{1}-\bigcup_{i \neq 1} A_{i}, 1 \in A_{m}-\bigcup_{i \neq m} A_{i}$, and $A_{i} \cap A_{j} \neq \varnothing$ if and only if $j=i-1, i$, or $i+1$. Choose $b(i) \in \Gamma_{a, r+1}, 0<b_{r+1}(i)<1$, and $\gamma>0$ so that the intervals $F_{i}=$ $\left[b_{r+1}(i)-\gamma, b_{r+1}(i)+\gamma\right]$ are mutually disjoint and $F_{i} \subset A_{i} \cap A_{i+1} \cap$ $(0,1)(i=1,2, \cdots, m-1)$.

Let $\Omega=\bigcup_{i, j, \sigma} \Omega_{c(i), j, \sigma}$. Since $B_{f} \cap \Omega=\varnothing$ (by $S_{r}$ (2) (iii) and (iv)), there is a $\delta$ with $0<\delta<\min \left(\varepsilon, d\left(B_{f}, \Omega\right)\right)(d$ is distance). Let $\alpha(b(\mathrm{i}))>$ $0, \mathfrak{I}(b(i))$, and $\left\{L_{b(i), j, o}\right\}$ be as given by $S_{r}$ for $\varepsilon$ replaced by $\delta$ and a replaced by $b(i)(i=1,2, \cdots, m-1)$; let $\beta=\min \{\alpha(b(i)), \alpha(c(i)), \gamma\}$. By $S_{r}$ (2) (vi) each $\operatorname{dim} L_{b(i), j}<\delta<d\left(B_{f}, \Omega\right)$ and by $S_{r}$ (2) (iv) $B_{f} \cap$ $L_{b(i), j} \neq \varnothing$; thus $\left(^{*}\right)$ if 


$$
\left(D^{2} \times \Gamma_{a, r+1}(r, \beta)\right) \cap L_{b(i), j, \sigma} \cap L_{c(h), k, \tau} \neq \varnothing,
$$

then $\left(D^{2} \times \Gamma_{a, r}(r, \beta)\right) \cap L_{b(i), j, \sigma} \subset\left(D^{2} \times \Gamma_{a, r}(r, \beta)\right) \cap\left(L_{c(h), k, \tau}-\Omega_{c(h), k, \tau}\right)$.

Let $d(t)(t=1,2, \cdots, 2 m)$ be the numbers $0,1, b_{r+1}(i)-\beta$, and $b_{r+1}(i)+\beta(i=1, \cdots, m-1)$ in increasing order. Then $\Psi(d(2 i-1)$, $d(2 i), \beta)$ (resp., $\Psi(d(2 i), d(2 i+1), \beta)$ ) is contained in $\Gamma_{c(i), r}(r, \alpha(c(i)))$ (resp., $\left.\Gamma_{b(i), r}(r, \alpha(b(i)))\right)$.

For each closed $r$-simplex $\sigma$ of $\mathfrak{I}(c(i))$ (resp., $\mathfrak{I}(b(i)))$, let $\Sigma \subset \Gamma_{a, r+1}$ be the closed $(r+1)$-cell defined by $x \in \Sigma$ if and only if $\left(x_{1}, \cdots, x_{r}\right.$, $\left.a_{r+1}, \cdots, a_{p-1}\right) \in \sigma, d(2 i-1) \leqq x_{r+1} \leqq d(2 i)$ (resp., $d\left(2_{i}\right) \leqq x_{r+1} \leqq d(2 i+$ $1)$ ), and $x_{i}=a_{i}$ for $i>r+1$. There is a triangulation $\mathfrak{I}$ of $\Gamma_{a, r+1}$ such that each such $\Sigma$ is a subpolyhedron [13, Chapter 1, p. 5]. For each closed $(r+1)$-simplex $\rho$ of $\mathfrak{T}$, there is an $r$-simplex $\sigma$ of $\mathfrak{T}(c(i))$ or $\mathfrak{I}(b(i))$ with $\rho \subset \Sigma$. Define $L_{j, \rho}=L_{j, \sigma} \cap\left(D^{2} \times \rho(r+1, \beta)\right)(j=1$, $2, \cdots, q(\rho)=q(\sigma))$. It follows that $S_{r+1}$ is satisfied for $\varepsilon$ and $a$, with $\beta>0, \mathfrak{I}$, and $\left\{L_{j, \rho}\right\}$ (conclusion (3) follows from (*) and $S_{r}(3)$ ).

Thus $S_{p-1}$ is true for(say) 0 and any $\varepsilon>0$; note that $\Gamma_{0, p-1}=I^{p-1}$ itself, and $\alpha$ does not arise in this case.

Let $e=1,2, \cdots$. Let $\mathfrak{I}_{e}$ be the triangulation of $I^{p-1}$ and let $\left\{L_{j, \sigma, e}\right\}$ be as given in $S_{p-1}$ for $\varepsilon=1 / e$, let $L_{e}=\mathbf{U}_{j, \sigma} L_{j, \sigma, e}$, and let $\Omega_{e}=$ $\bigcup_{j, \sigma} \Omega_{j, \sigma, e}$. Each $\mathfrak{T}_{e}$ is rectilinear in $I^{p-1}$, so we may suppose that each $\mathfrak{T}_{e+1}$ is a subdivision of $\mathfrak{I}_{e}$.

Define an equivalence relation $\sim$ on $L_{e}$ by: for every $a \in I^{p-1}, \sigma$, and $j$, and for every $u, v \in L_{j, \sigma, e} \cap\left(D^{2} \times\{a\}\right), u \sim v$. Let $Y_{e}$ be the resulting identification space, and let $\omega_{e}: L_{e} \rightarrow Y_{e}$ be the identification map. Let $L_{e} \cap\left(D^{2} \times \partial I^{p-1}\right)$ be denoted by $G_{e}$, and $\omega_{e}\left(G_{e}\right)$ by $\partial Y_{e}$. Then $\omega_{e}:\left(L_{e}, G_{e}\right) \rightarrow\left(Y_{e}, \partial Y_{e}\right)$ is a homotopy equivalence, $Y_{e}$ is a $(p-1)$ dimensional finite polyhedron, viewed as a cell complex [13, Chapter 1 , p. 5], its closed $(p-1)$-cells are $\omega_{e}\left(L_{j, a, e}\right)$, their interiors $\omega_{e}\left(L_{j, a, e} \cap\right.$ $\left.\left(D^{2} \times \operatorname{int} \sigma\right)\right)=\gamma_{j, \sigma, e}$ are mutually disjoint for distinct pairs $(j, \sigma)$.

With the index $\xi$ of $[7,(2.1)] \sum_{j, \sigma} \xi\left(L_{j, \sigma, e}\right) \cdot \gamma_{j, \sigma, e}$ is a $(p-1)$-chain $\beta_{e}$ of $\left(Y_{e}, \partial Y_{e}\right)$. From the index formula [7, (2.3)] and from (2) (v) and (3) in $S_{p-1}$ (note that $\mathrm{Cl}[(\sigma \cap \tau)(\alpha)]$ is merely $\sigma \cap \tau$ in this case), it follows that $\beta_{e}$ is a cycle of $\left(Y_{e}, \partial Y_{e}\right)$. Since $\xi\left(D^{2} \times\{s\}\right)=1$, it follows again from the index formula that $\sum_{j} \xi\left(L_{j, \sigma}\right)=1$ for each $\sigma$, so that $\beta_{e} \neq 0$. Since $\operatorname{dim} Y_{e}=p-1, \beta_{e}$ defines a nonzero element of $H_{p-1}\left(Y_{e}, \partial Y_{e} ; Z\right) \approx H_{p-1}\left(L_{e}, G_{e}, Z\right)(Z$ the ring of integers). Let $\eta_{e}=\omega_{e *}^{-1}\left(\left\{\beta_{e}\right\}\right) \in H_{p-1}\left(L_{e}, G_{e} ; Z\right)$.

Since $\Omega_{e} \cap B_{f}=\varnothing$ (by $S_{p-1}$ (2) (iv)), there exists $\delta(e)$ with $0<$ $\delta(e)<d\left(\Omega_{e}, B_{f}\right)(e=1,2, \cdots)$, and there is a subsequence $\{e(k)\}$ such that $e(1)=1$ and $1 / e(k+1)<\min \{\delta(e(i)): i \leqq k\}(k=1,2, \cdots)$. For every $L_{j, \sigma, e(k+1)}$, there are a unique $\tau \in T_{e(k)}$ with $\sigma \subset \tau$ and $x \in B_{f} \cap$ $L_{j, \sigma, e(k+1)}$ by $S_{p-1}(2)$ (iv). For a unique $i, x \in L_{i, \tau, e(k)}$ by $S_{p-2}$ (2) (iv) and $(V)$, and from the size of $1 / e(k+1)$ and $S_{p-1}$ (2) (vi), (†) $L_{j, \sigma, e(k+1)} \subset$ 
$L_{i, \tau, e(k)}$. Let $\lambda_{k+1}:\left(L_{e(k+1)}, G_{e(k+1)}\right) \rightarrow\left(L_{e(k)}, G_{e(k)}\right)$ be inclusion. From ( $\left.\dagger\right)$ and the index formula $[7,(2.3)]$ it follows that $\lambda_{k+1}^{*}\left(\eta_{e(k+1)}\right)=\eta_{e(k)}(\neq 0)$. Thus the inverse limit of $\left\{\eta_{e(k)}\right\}$ is nonzero, so that the Cech homology group $H_{p-1}\left(\bigcap_{e} L_{e}, \bigcap_{e} G_{e} ; Z\right) \neq 0$ by the Continuity Theorem. Hence

$$
\operatorname{dim}\left(\bigcap_{e} L_{e}\right) \geqq p-1
$$

[9, p. 152, Theorem VIII 4], and since $\bigcap_{e} L_{e} \subset B_{f}\left(S_{p-1}\right.$ (2) (iv) and (vi)), $\operatorname{dim} B_{f} \geqq p-1$.

3. Differential lemmas. The following two lemmas are generalizations of lemmas that have been used repeatedly, and these generalizations will also be used elsewhere.

LEMma 3.1. Let $f: M^{n} \rightarrow N^{p}$ be $C^{m}$, let $K^{q}$ be a $C^{m} q$-manifold $(m=1,2, \cdots$; or $m=\infty$; or $m=\omega ; q=0,1, \cdots, p-1)$, let $\rho$ be $a$ $C^{m}$ diffeomorphism of a region in $N^{p}$ onto $K^{q} \times R^{p-q}$, and let $\Omega$ be a nonempty compact subset of $f^{-1}\left(\rho^{-1}\left(K^{q} \times\{0\}\right)\right)$. If $f \mid \Omega$ is transverse regular on $\rho^{-1}\left(K^{q} \times\{0\}\right)$, then there are $\varepsilon>0, a C^{m}(n-p+q)$-manifold $L$, and $a C^{m}$ diffeomorphism $\sigma$ of $L \times S(0, \varepsilon)$ onto a neighborhood of $\Omega$ in $M^{n}$ such that $\rho \circ f \circ \sigma$ is a layer map.

This is proved in $[6,(4.1)]$ and is a generalization of $[8$, p. 80 , (3.5)] and [3, p. 376, (2.7)]. The condition that " $f \mid \Omega$ is transverse regular" means that $f$ is transverse regular at $x$ for each $x \in \Omega$.

Lemma 3.2. Let $q=1,2, \cdots$, let $f: M^{n} \rightarrow N^{p}$ be a $C^{r}$ map with $\max (n-q+1,1) \leqq r \leqq \infty$, let $\Omega \subset M^{n}$ be compact, and let $Y \subset N^{p}$ be closed, with $\operatorname{dim} Y \geqq q$. Then for some $m(m=0,1, \cdots, p-q)$ there is a $C^{r}$ embedding $\lambda$ of $S^{m} \times R^{p-m}$ in $N^{p}$ such that $f \mid \Omega$ is transverse regular on $\lambda\left(S^{m} \times\{t\}\right)$ and $\lambda\left(S^{m} \times\{t\}\right) \cap Y \neq \varnothing$ for each $t \in R^{p-m}$.

If $\Omega$ is omitted, " $f \mid \Omega$ is transverse regular" is replaced by " $f$ is transverse regular", and $f$ is assumed proper, this is $[8$, p. 80, (3.7)]. The proof is an immediate generalization of that proof. (Although we do not need it in this paper, the same comments apply to $[8$, p. 82 , (3.8)], except that $J$ need not be compact.)

DEFINITION 3.3. Let $K^{n}$ and $L^{p}$ be $C^{r}$-manifolds with nonempty boundary, and let $f: K^{n} \rightarrow L^{p}$ be a $C^{r}(r \geqq 1)$ proper map with $f^{-1}\left(\partial L^{p}\right)=$ $\partial K^{p}$ and $f\left(R_{p-1}(f)\right) \subset \operatorname{int} L^{p}$. Let $D\left(K^{n}\right)$ and $D\left(L^{p}\right)$ be the doubles $K^{n}$ and $L^{p}$, respectively $[10$, p. $52,(5.10)$ and p. 62, (6.3)]. We now define a $C^{r}$ map $g: D\left(K^{n}\right) \rightarrow D\left(L^{p}\right)$, called a double of $f$, such that the restriction of $g$ to each half is $C^{r}$ equivalent to $f[5, \mathrm{p} .616$, 
(1.3)].

Let $K_{i}=K \times i$, let $L_{i}=L \times i$, and let $f_{i}: K_{i} \rightarrow L_{i}$ be defined by $f_{i}(x, i)=(f(x), i)(i=0,1)$. Let $J_{0}=[0,1)$ and $J_{1}=(-1,0]$. There is an open neighborhood $U$ of $\partial L$ in $L$ disjoint from $f\left(R_{p-1}(f)\right)$ and $C^{r}$ diffeomorphisms $\psi_{i}: U_{i}=U \times i \rightarrow \partial L_{i} \times J_{i}[10$, p. 51, (5.9)]. Let $\alpha_{i}: f_{i}^{-1}\left(U_{i}\right) \rightarrow U_{i}$ and $\beta_{i}: \partial K_{i} \rightarrow \partial L_{i}$ be the restrictions of $f_{i}$.

There exist manifolds $V_{i}=V_{i}^{n}$ with $\partial V_{i}=\varnothing$ and $f^{-1}\left(U_{i}\right) \subset V_{i}$ and $W_{i}=W_{i}^{p}$ with $\partial W_{i}=\varnothing$ and $U_{i} \subset W_{i}$, and a $C^{r}$ extension $\gamma_{i}: V_{i} \rightarrow$ $W_{i}$ of $\alpha_{i}$. By restricting $\gamma_{i}$ we may suppose that it is proper. Now $\gamma_{i}$ is the projection map of a $C^{r}$ bundle (e.g. from (3.1) with $K$ a single point), so that $\alpha_{i}$ and $\beta_{i}$ are also. Thus there are diffeomorphisms $\phi_{i}: f_{i}^{-1}\left(U_{i}\right) \rightarrow \partial K_{i} \times J_{i}$ such that $\psi_{i} \circ \alpha_{i}=\left(\beta_{i} \times \iota\right) \circ \phi_{i}$ (where $\iota$ is the identity map on $J_{i}$ ) [11, p. 53, (11.4)].

We may define the $\left(C^{r}\right.$ structures on the) doubles $D\left(K^{n}\right)$ and $D\left(L^{p}\right)$ using the maps $\phi_{i}$ and $\psi_{i}$ (identify $(x, 0)$ in $\partial K_{0}$ with $(y, 1)$ in $\partial K_{1}$ if $\phi_{0}(x, 0)$ and $\phi_{1}(y, 1)$ have the same first coordinate), and let $\lambda_{i}$ : $K_{i} \rightarrow D\left(K^{n}\right)$ and $\mu_{i}: L_{i} \rightarrow D\left(L^{p}\right)$ be the natural $\left(C^{r}\right)$ embeddings. Define $g$ by $g(x)=f_{i}(x)$ for $x \in K_{i}$. Clearly $g$ is $C^{r}$ except possibly on $\partial K$. If $U^{\prime}=U_{0} \cup U_{1}$ and $\psi: U^{\prime} \rightarrow \partial L \times(-1,1)$ and $\phi: g^{-1}(U) \rightarrow \partial K \times(-1.1)$ are defined by the $\psi_{i}$ and $\phi_{i}$, respectively, then $\psi \circ g \mid g^{-1}\left(U^{\prime}\right)=(\beta \times \iota) \circ \phi$ (where $c$ is the identity map on $(-1,1)$ and $\beta=\beta_{1}=\beta_{2}$ ), so that $g$ is $C^{r}$ everywhere.

LeMma 3.4. Let $f: M^{n} \rightarrow N^{p}$ be a $C^{n}$ map with $n-p=0$ or 1 , $\operatorname{dim} B_{f} \leqq p-2$, and $\operatorname{dim}\left(B_{f} \cap f^{-1}(y)\right) \leqq 0$ for each $y \in N^{p}$. Then $f$ is open.

Proof. In case $n=p, f$ is light and the conclusion is given by $[2$, p. $94,(2.3)]$, so we may suppose that $n=p+1$. Suppose that $f$ is not open. Let $E_{f}$ be the set of points at which $f$ fails to be open, and let $x \in E_{f}$. According to [5, p. 622, (2.6)] there is a connected (not necessarily compact) manifold $K^{p+1} \subset M^{p+1}$ with boundary such that $x \in \operatorname{int} K^{p+1}\left(=K^{p+1}-\partial K^{p+1}\right)$ and the closure $\bar{K}^{p+1}$ of $K^{p+1}$ in $M^{p+1}$ is compact; there is an open $p$-cell $D^{p} \subset N^{p}$ with $f\left(K^{p+1}\right) \subset D^{p}$; and the restriction map $g: K^{p+1} \rightarrow D^{p}$ is proper with $B_{g} \cap \partial K^{p+1}=\varnothing$. Let $\psi=g \mid$ int $K^{p+1}$, and let $\Omega \subset$ int $K^{p+1}$ be the compact set $E_{\psi}$. Since $f$ is not open, $\operatorname{dim} \psi\left(E_{\psi}\right) \geqq p-1[5$, p. 623, (3.4)], and by (3.2) there is a $C^{p+1}$ embedding $\lambda: S^{m} \times R^{p-m} \rightarrow D^{p}$ such that $\psi \mid \Omega$ is transverse regular on $\lambda\left(S^{m} \times\{t\}\right)$ and $\lambda\left(S^{m} \times\{t\}\right) \cap \psi\left(E_{\psi}\right) \neq \varnothing$ for each $t \in R^{p-m}$ and $m=0$ or 1 . From (3.1) $m \neq 0$ and, for some $\varepsilon>0$, the restriction of $\psi$ to some neighborhood of $E_{\psi}$ is $C^{p+1}$ equivalent to the $C^{p+1}$ layer map $\alpha: Q^{2} \times R^{p-1} \rightarrow S^{1} \times R^{p-1}$ with $E_{\alpha} \cap\left(Q^{2} \times\{t\}\right) \neq \varnothing$ for every $t \in R^{p-1}$.

Since $B_{\alpha} \subset R_{p-1}(\alpha)$ (the Rank Theorem [5, p. 617, (1.6)], $\operatorname{dim}\left(\alpha\left(B_{\alpha}\right) \cap\right.$ 
$\left.\left(S^{1} \times\{t\}\right)\right) \leqq 0$ for each $t \in R^{p-1}$ (by Sard's theorem); and since

$$
\operatorname{dim}\left(B_{\alpha} \cap \alpha^{-1}(u, t)\right) \leqq 0
$$

for each $(u, t) \in S^{1} \times R^{p-1}$ by hypothesis, $\operatorname{dim}\left(B_{\alpha} \cap\left(Q^{2} \times\{t\}\right)\right) \leqq 0[9$, p. 91, Theorem VI 7].

Let $(q, s) \in E_{\alpha} \subset B_{\alpha}$ (we may suppose that $s=0$ ), and let $T \subset Q^{2} \times$ $R^{p-1}$ be a closed $(p+1)$-cell neighborhood of $(q, 0)$. Since $\{(q, 0)\}$ is the component of $\alpha^{-I}(\alpha(q, 0))$ containing $(q, 0)$ [5, p. 622, (3.2)], there is an interval $I \subset S^{1}$ with $\alpha_{0}(q) \in \operatorname{int} I$ and $\delta>0$ such that the component $F$ of $\alpha^{-1}(I \times S(s, \delta))$ is contained in int $T$. We may suppose that the endpoints of $I$ are regular values of $\alpha_{0}$, and thus, for $\delta$ sufficiently small, of $\alpha_{t}$ for every $t \in S(0, \delta)$. Thus $F$ is an $n$-manifold with boundary, and each $F_{t}=F \cap\left(Q^{2} \times\{t\}\right)$ is compact. Let $G$ be the double of $F$, and let $\beta: G \rightarrow S^{1} \times S(0, \delta)$ be the double of the proper $\operatorname{map} \alpha \mid F: F \rightarrow I \times S(0, \delta)(3.3)$.

Choose an open 2-cell $U$ with $q \in U$ and $U \times\{0\} \subset$ int $F_{0} \subset G_{0}$, and choose $\eta, 0<\eta<\delta$, with $U \times S(0, \eta) \subset \operatorname{int} F \subset G$. There exists $\xi, 0<$ $\xi<\eta$, and an interval $J \subset$ int $I \subset S^{1}$ such that $\beta_{0}(q) \in \operatorname{int} J$, the component $X$ of $\beta^{-1}(J \times S(0, \xi))$ containing $(q, 0)$ is contained in $U \times S(0, \xi)$, and the end points of $J$ are regular values of $\beta_{t}$ for each $t \in S(0, \xi)$. Thus $X \cap(U \times\{0\})$, call it $A^{2}$, is a 2-disk with holes, and $\alpha_{0}\left(\partial A^{2}\right) \subset \partial J$.

We now apply [1, p. 196, (3.4)] to $\beta, K_{0}=S^{1} \times\{0\}, \Gamma_{1}=J \times\{0\}$, $K_{1}=\partial \Gamma_{1}$, and $\rho$ the identity map. There exists $\zeta, 0<\zeta<\xi$, and a $C^{p+1}$ (layer) diffeomorphism $\omega$ of $\beta^{-1}\left(S^{1} \times\{0\}\right) \times S(0, \zeta)$ onto $\beta^{-1}\left(S^{1} \times\right.$ $S(0, \zeta))$ with $\omega\left(A^{2} \times S(0, \zeta)\right)=X$. Let $D$ be the closed 2-cell with $A^{2} \subset D \subset U$ and $\partial D \subset \partial A^{2}$, and let $\gamma: D \times S(0, \zeta) \rightarrow \operatorname{int} I \times S(0, \zeta)$ be the restriction of $\beta \circ \omega$. Now $(0, q) \in E_{\gamma} \subset B_{\gamma}$ and by (2.1) $\operatorname{dim} B_{r}=p-1$, so that $\operatorname{dim} B_{f} \geqq p-1$, and a contradiction results.

\section{Conclusions.}

Proposition 4.1. Let $f: M^{p+1} \rightarrow N^{p}$ be $C^{p+1}$ with $B_{f} \neq \varnothing, \operatorname{dim} B_{f} \leqq$ $p-2$, and $\operatorname{dim}\left(f^{-1}(y) \cap B_{f}\right) \leqq 0$ for each $y \in N^{p}$. Then $\operatorname{dim} B_{f}=$ $p-3$ and there is a closed set $Y \subset B_{f}$ such that $\operatorname{dim} Y<p-3$ and, for every $x \in B_{f}-Y, f$ at $x$ is locally topologically equivalent to

$$
\tau \times \mathrm{id}: R^{4} \times R^{p-3} \longrightarrow R^{3} \times R^{p-3} .
$$

According to the Rank Theorem [5, p. 617, (1.6)] $B_{f} \subset R_{p-1}(f)$ and the following corollary results.

CoROLlaRY 4.2. Let $f: M^{p+1} \rightarrow N^{p}$ be $C^{p+1}$ with critical set $R_{p-1}(f)$, let $\operatorname{dim} R_{p-1}(f) \leqq p-2$, and let $\operatorname{dim}\left(f^{-1}(y) \cap R_{p-1}(f)\right) \leqq 0$ for each $y \in N^{p}$. Then there is a closed set $Y \subset M^{p+1}$ such that $\operatorname{dim} Y<p-3$ 
and, for each $x \in M^{p+1}-Y, f$ at $x$ is locally topologically equivalent to either the projection map $\rho: R^{p+1} \rightarrow R^{p}$ or to

$$
\tau \times \mathrm{id}: R^{4} \times R^{p-3} \longrightarrow R^{3} \times R^{p-3} .
$$

Proof of (4.1). By (3.4) $f$ is open, and $p \geqq 2$ since $B_{f} \neq \varnothing$ and $\operatorname{dim} B_{f} \leqq p-2$. According to [7, (4.1) and (1.1)], if $f: M^{p+1} \rightarrow N^{p}$ is a $C^{3}$ open map with $\operatorname{dim}\left(B_{f} \cap f^{-1}(y)\right) \leqq 0$ for each $y \in N^{p}$, then there is a closed set $X \subset M^{p+1}$ such that $\operatorname{dim} f(X) \leqq p-2$ and, for every $x \in M^{p+1}-X$, there is a natural number $d(x)$ with $f$ at $x$ locally topologically equivalent to the map

$$
\phi_{d(x)}: C \times R^{p-1} \longrightarrow R \times R^{p-1}
$$

defined by $\phi_{d^{(x)}}(z, t)=\left(\mathscr{R}\left(z^{d(x)}\right), t\right)\left(\mathscr{R}\left(z^{d(x)}\right)\right.$ is the real part of the complex number).

Since $\operatorname{dim} B_{f} \leqq p-2$ by hypothesis, $B_{f} \subset X$, so that $\operatorname{dim} f\left(B_{f}\right) \leqq$ $p-2$. Thus $f$ satisfies the hypothesis of $[5$, p. 626, (4.7)]. (For $n=p+1$ that proposition is identical with the present one except that the hypothesis $\operatorname{dim} B_{f} \leqq p-2$ is replaced by $\operatorname{dim} f\left(B_{f}\right) \leqq p-2$.)

CoRollary 4.3. If $f: M^{p+1} \rightarrow N^{p}$ is a $C^{p+1}$ map with $\operatorname{dim} B_{f}=$ 0 and $p \geqq 2$, then $p=3$ and at each $x \in B_{f}, f$ is locally topologically equivalent to $\tau$.

4.4. Proof of (1.1). From the Rank Theorem [5, p. 617, (1.6)] $B_{f} \subset R_{p-1}(f)$, and the conclusion for $n-p=1$ results from (4.3). For $n=p \geqq 3 \operatorname{dim}\left(R_{p-1}(f)\right) \leqq 0$ implies $B_{f}=\varnothing[2$, p. 94, (2.2)]; for $n=$ $p=2, f$ is light open [2, p. $94,(2.3)]$, and so has the desired structure (e.g. by $[2$, p. $90,(1.10)]$ ).

Let $G$ be a compact, connected Lie group, and let $M$ be a closed, connected, oriented $G$-manifold with orbit space a manifold. The action is called almost free if it is free except for the fixed point set $F$, and $F$ is discrete nonempty set. In [4] Church and Lamotke classified such actions globally, up to equivariant homeomorphism (they also treated the smooth case): invariants are the oriented homeomorphism type of the orbit space and the number (which is even) of fixed points. This classification gives significance to the following corollary of (1.1), a global classification of maps with 0 -dimensional critical set.

CoRollary 4.5. Let $M^{p+1}$ and $N^{p}$ be closed, connected, oriented manifolds, and let $f: M^{p+1} \rightarrow N^{p}$ be a $C^{p+1}$ map with critical set $R_{p-1}(f)$ of dimension at most 0 . Then there is a unique factorization $f=h \circ g$, where $g: M^{p+1} \rightarrow K^{p}$ is the orbit map of a topological $S^{1}$ free or almost free action on $M^{p+1}$ (and thus is classified by [4]), 
and $h: K^{p} \rightarrow N^{p}$ is an $r$-to-1 covering $\operatorname{map}(r=1,2, \cdots)$.

Proof. By (1.1) either the branch set $B_{f}=\varnothing$, or $p=3$ and at each point of $B_{f} f$ is locally topologically equivalent to $\tau$, i.e., to the cone map of the Hopf fibration $\psi: S^{3} \rightarrow S^{2}$ [5, p. 618, (1.10)]. According to $[12$, p. $64,(2.5)]$ there is a natural number $k$ such that $f^{-1}(y)$ has exactly $k$ components for each $y \in N^{p}-f\left(B_{f}\right)$, and at most $k$ components for each $y \in f\left(B_{f}\right)$. From the local structure, $f^{-1}(y)$ has exactly $k$ components for every $y \in N^{p}$, and thus according to $[12$, p. $63,(2.1)]$ there is a (unique) factorization $f=h \circ g$, where $g: M^{p+1} \rightarrow K^{p}$ is a $C^{p+1}$ monotone map and $h: K^{p} \rightarrow N^{p}$ is an $r$-to-1 covering map.

In case $B_{f}=\varnothing, B_{g}=\varnothing$ also, so that $g$ is a bundle map [5, p. $618,(1.9)]$ with fiber $S^{1}$. The structure group can be reduced to $S^{1}=$ $S O(2)$ [12, pp. 64-65], and thus $g$ is the orbit map of a free $S^{1}$ action. In case $B_{f} \neq \varnothing$, the map $\alpha: M^{p+1}-B_{g} \rightarrow K^{p}-g\left(B_{g}\right)$ defined by restriction of $g$ is also a free $S^{1}$ action; since $B_{g}$ is discrete, $g$ itself is the orbit map of an almost free action.

\section{REFERENCES}

1. P. T. Church, Differentiable monotone maps on manifolds, Trans. Amer. Math. Soc., 128 (1967), 185-205.

2. Differential open maps on manifolds, Trans. Amer. Math. Soc., 109 (1963), 87-100.

3. - Factorization of differentiable maps with branch set dimension at most $n-3$, Trans. Amer. Math. Soc., 115 (1965), 370-387.

4. P. T. Church and K. Lamotke, Almost free G-manifolds, Bull. Austal. Math. Soc., 10 (1974), 171-196.

5. P. T. Church and J. G. Timourian, Differentiable maps with 0-dimensional critical set, I, Pacific J. Math., 41 (1972), 615-630.

6. Differentiable maps with 0-dimensional set, II, Indiana U. Math. J., 24 (1974), 17-25.

7. Differentiable open maps from $(p+1)$-manifold to $p$-manifold, Pacific J. Math., 48 (1973), 35-45.

8. - Fiber bundles with singularities, J. Math. Mech., 18 (1968), 71-90.

9. W. Hurewicz and H. Wallman, Dimension Theory, 2nd edition, Princeton University Press, Princeton, 1948.

10. J. R. Munkres, Elementary Differential Topology, Princeton University Press, Princeton, 1963.

11. N. Steenrod, The Topology of Fiber Bundles, Princeton University Press, Princeton, 1951.

12. J. G. Timourian, Fiber bundles with discrete singular set, J. Math. Mech., 18 (1968), 61-70.

13. E. C. Zeeman, Seminar on Combinatorial Topology, Institute des Haûtes Etudes Scientifiques, mimeographed, Paris, 1963.

Received February 7, 1973. Work of the first author supported by N.S.F Grant GP-6871 and that of the second author by N. S. F. Grant GP-8888 and NRC Grant A 7357.

SYRACUSE UNIVERSITY

AND

UnIVERSity OF ALberta 


\title{
PACIFIC JOURNAL OF MATHEMATICS
}

\author{
EDITORS
}

RICHARD ARENS (Managing Editor)

University of California

Los Angeles, California 90024

R. A. Beaumont

University of Washington

Seattle, Washington 98105
J. DugundJI

Department of Mathematics University of Southern California Los Angeles, California 90007

D. Gilbarg and J. Milgram

Stanford University

Stanford, California 94305

\section{ASSOCIATE EDITORS}

E. F. BECKENBACH

B. H. NeUManN

F. WOLF

K. YosHIDA

\section{SUPPORTING INSTITUTIONS}

UNIVERSITY OF BRITISH COLUMBIA

GALIFORNIA INSTITUTE OF TECHNOLOGY

UNIVERSITY OF CALIFORNIA

MONTANA STATE UNIVERSITY

UNIVERSITY OF NEVADA

NEW MEXICO STATE UNIVERSITY

OREGON STATE UNIVERSITY

UNIVERSITY OF OREGON

OSAKA UNIVERSITY
UNIVERSITY OF SOUTHERN CALIFORNIA

STANFORD UNIVERSITY

UNIVERSITY OF TOKYO

UNIVERSITY OF UTAH

WASHINGTON STATE UNIVERSITY

UNIVERSITY OF WASHINGTON

AMERICAN MATHEMATICAL SOCIETY NAVAL WEAPONS CENTER 


\section{Pacific Journal of Mathematics}

\section{Vol. 57, No. $1 \quad$ January, 1975}

Keith Roy Allen, Dendritic compactification ......................... 1

Daniel D. Anderson, The Krull intersection theorem ..................... 11

George Phillip Barker and David Hilding Carlson, Cones of diagonally dominant

matrices .............................................

David Wilmot Barnette, Generalized combinatorial cells and facet splitting ........

Stefan Bergman, Bounds for distortion in pseudoconformal mappings ............

Nguyên Phuong Các, On bounded solutions of a strongly nonlinear elliptic

equation ...............................................

Philip Throop Church and James Timourian, Maps with 0-dimensional critical

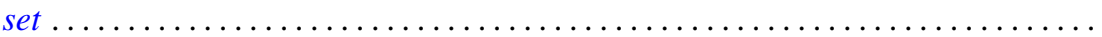

G. Coquet and J. C. Dupin, Sur les convexes ubiquitaires ................. 67

Kandiah Dayanithy, On perturbation of differential operators............... 85

Thomas P. Dence, A Lebesgue decomposition for vector valued additive set

functions .............................................. 91

John Riley Durbin, On locally compact wreath products.................. 99

Allan L. Edelson, The converse to a theorem of Conner and Floyd ............. 109

William Alan Feldman and James Franklin Porter, Compact convergence and the

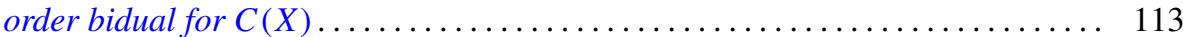

Ralph S. Freese, Ideal lattices of lattices......................... 125

R. Gow, Groups whose irreducible character degrees are ordered by divisibility ... 135

David G. Green, The lattice of congruences on an inverse semigroup ............ 141

John William Green, Completion and semicompletion of Moore spaces .......... 153

David James Hallenbeck, Convex hulls and extreme points of families of starlike and close-to-convex mappings .................................. 167

Israel (Yitzchak) Nathan Herstein, On a theorem of Brauer-Cartan-Hua type ...... 177

Virgil Dwight House, Jr., Countable products of generalized countably compact spaces ............................................ 183

John Sollion Hsia, Spinor norms of local integral rotations. I . . . . . . ......... 199

Hugo Junghenn, Almost periodic compactifications of transformation

semigroups....................................

Shin'ichi Kinoshita, On elementary ideals of projective planes in the 4-sphere and oriented $\Theta$-curves in the 3 -sphere ............................ 217

Ronald Fred Levy, Showering spaces............................ 223

Geoffrey Mason, Two theorems on groups of characteristic 2-type............. 233

Cyril Nasim, An inversion formula for Hankel transform ................... 255

W. P. Novinger, Real parts of uniform algebras on the circle ................ 259

T. Parthasarathy and T. E. S. Raghavan, Equilibria of continuous two-person

games............................................... 265

John Pfaltzgraff and Ted Joe Suffridge, Close-to-starlike holomorphic functions of several variables ....................................... 271

Esther Portnoy, Developable surfaces in hyperbolic space .................. 281

Maxwell Alexander Rosenlicht, Differential extension fields of exponential type . . . 289

Keith William Schrader and James Lewis Thornburg, Sufficient conditions for the

existence of convergent subsequences ........................... 301

Joseph M. Weinstein, Reconstructing colored graphs .................. 307 